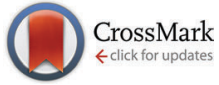

Cite this: J. Mater. Chem. C, 2016, 4, 2187

Received 25th December 2015, Accepted 13th February 2016

DOI: $10.1039 / c 5 t c 04388 c$

www.rsc.org/MaterialsC

\title{
The pivotal step of nanoparticle functionalization for the preparation of functional and magnetic hybrid opal films $\dagger$
}

\author{
D. Scheid, ${ }^{a}$ D. Stock, ${ }^{a}$ T. Winter, ${ }^{a}$ T. Gutmann, ${ }^{{ }^{b}}$ C. Dietz ${ }^{\mathrm{cd}}$ and M. Gallei*a
}

\begin{abstract}
The preparation of hierarchical and sophisticated particle architectures for mimicking structural colors known from nature still remains a challenge. In this study, the preparation of novel opal and doubleinverse opal films based on thermally treated metallopolymer core particles with a silica shell is described. Thermal treatment leads to the formation of magnetic nanorattle-type particles. The main challenge of artificial particles is to ensure sufficient dispersibility after several synthetic steps. Especially silica particles providing surface hydroxyl groups tend to sinter at high temperatures leading to agglomeration. We present the introduction of trimethyl ethoxy silane (TMES) as an excellent functionalization reagent as the key reaction step. The necessity and success of functionalization are investigated by transmission electron microscopy (TEM) and zeta potential measurements. Importantly, solid state NMR techniques are employed to gain deeper insights into the chemical structure of the surface-attached reagent. Finally, by this convenient functionalization the preparation of elastomeric opal films and double-inverse opal films is proven successful revealing excellent optical film properties. Moreover, magnetic properties of these novel films are investigated by using magnetic force microscopy (MFM). The herein established route is expected to pave the way for the preparation of a variety of advanced and stimuli-responsive optical materials.
\end{abstract}

\section{Introduction}

Self-assembly of colloidal particles consisting of polymeric as well as inorganic materials in a crystalline manner leads to photonic band gap materials for various potential applications, for instance, optoelectronic sensing devices and actuation systems. ${ }^{1}$ Due to the high order and periodicities in the range

\footnotetext{
${ }^{a}$ Ernst-Berl Institut für Technische und Makromolekulare Chemie, Technische Universität Darmstadt, Alarich-Weiss-Str. 4, D-64287 Darmstadt, Germany.E-mail: M.Gallei@mc.tu-darmstadt.de

${ }^{b}$ Eduard-Zintl Institut für Anorganische und Physikalische Chemie, Technische Universität Darmstadt, Alarich-Weiss-Str. 8, D-64287 Darmstadt, Germany

${ }^{c}$ Center of Smart Interfaces and Department of Materials Science, Technische Universität Darmstadt, Alarich-Weiss-Str. 16, D-64287 Darmstadt, Germany

${ }^{d}$ Physics of Surfaces, Institute of Materials Science Department, Technische Universität Darmstadt, Alarich-Weiss-Str. 10, D-64287 Darmstadt, Germany

$\dagger$ Electronic supplementary information (ESI) available: Additional data on the synthesis scheme, images of particle dispersion, zeta potential measurements, thermogravimetric analysis, ${ }^{13} \mathrm{C}$ CP MAS spectra, description of silica anchoring groups, TEM images, $T_{1}$ relaxation times for ${ }^{29} \mathrm{Si}$, ${ }^{29} \mathrm{Si} \mathrm{CP}$-MAS spectra, calculations of reflected wavelengths of nanorattle-type films, and SEM images. See DOI: $10.1039 / \mathrm{c} 5 \mathrm{tc} 04388 \mathrm{c}$
}

of visible light, structural colors can be observed similar to optical effects known in a variety of animals, plants, and minerals. Therefore, artificial colloidal crystals have received a great deal of attention due to their fascinating, non-photodegradable, and energy saving properties, which are important for developing unprecedented coloring of materials. ${ }^{2-6}$ Inverse opal structures featuring an outstanding structural porosity have attracted enormous attention in the field of photonic bandgap materials and - due to their adjustable porosity - for many other applications comprising membranes and electrode materials. ${ }^{7-11}$ Opal films can be stimuli-responsive and the stop-band of the material can be influenced by several external triggers, e.g. light, solvents, temperature, ionic strength, redox chemistry or by applying an electrical field. ${ }^{12-16}$ Exemplarily, soft polymer-based inverse opal films can reveal excellent stretch-tunable properties and solvent responsiveness accompanied by photonic band gap switching. ${ }^{17}$ Electrically tunable colloidal structures as feasible materials for display technologies were reported by Lee et al. ${ }^{18}$ Here, the authors took advantage of amorphous $\mathrm{Fe}_{3} \mathrm{O}_{4} @ \mathrm{SiO}_{2}$ particles dispersed in propylene carbonate, revealing tunable structural colors across the visible region of 490 to $655 \mathrm{~nm}$, simply by applying a voltage. As another non-invasive and non-diffusion controlled stimulus, magnetism in photonic materials has been intensively investigated in the last decade. 


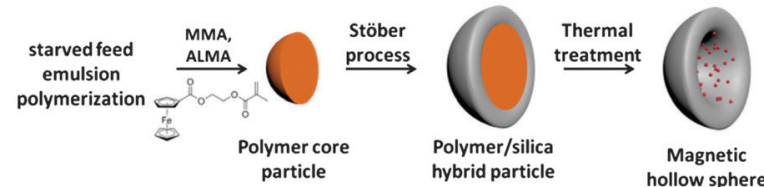

Fig. 1 Combination of emulsion polymerization, the Stöber process and calcination for the preparation of monodisperse magnetic nanoparticles.

Asher et al. investigated the magnetically induced crystallization of a polystyrene (PS) latex, containing superparamagnetic nanoparticles. ${ }^{19}$ Chen et al. took advantage of magnetically responsive colloidal particles for unforgeable watermarks on bank notes. ${ }^{20}$ Most prominent examples in the field of magnetically triggered photonic structures are reported by Yin and Ge in several publications, including the preparation of rewritable photonic paper or magnetochromic thin film plates. ${ }^{21-24}$ Recently, our group reported the preparation of magnetic (nanorattle-type) and magnetic hollow particles. ${ }^{25}$ For this purpose, a combination of starved feed emulsion polymerization of a ferrocene-containing monomer and the Stöber process leads to magnetic silica hollow spheres with adjustable diameters after thermal treatment (Fig. 1).

However, many approaches towards the industrial scale production of switchable opal materials come with issues. Typically, structures featuring a significant amount of defects are obtained during processing. Recently, the melt-shear organization technique was described as a promising tool for ordering inorganic core particles featuring a comparably soft polymeric shell. ${ }^{17,26}$ This technique, which enables the preparation of free-standing opal films with film diameters of $15 \mathrm{~cm}$ without the necessity of a particle dispersion medium, has not been reported for more complex particle architectures, for instance hollow, magnetic or nanorattle-type spheres.

In the present study we introduce a universal surface modification protocol for (complex and simple) silica particles by taking advantage of trimethyl ethoxy silane (TMES) as the functionalizing reagent. The functionalized hollow silica and magnetic core shell particles are investigated with respect to chemical functionality, stability in dispersion, as single source ceramic precursors and finally for the transfer into emulsion polymerization. The necessity and success of functionalization are investigated by transmission electron microscopy (TEM) and zeta potential measurements. Importantly, solid state NMR techniques are employed to gain deeper insights into the chemical structure of surface-attached reagents. The novel magnetic particles are used for the preparation of easy-scalable magnetic opal films and double-inverse opal films by using (i) the melt-shear organization technique or (ii) the vertical deposition method. Magnetic properties are investigated by magnetic force microscopy (MFM) measurements. Due to their convenient easy-scalable preparation, we expect that these magnetic opal films have potential as next generation optoelectronic and membrane materials. Moreover, with insights into the crucial step of silica modification, the herein presented route can be expanded to a wide range of functional core-shell architectures.

\section{Results and discussion}

\section{Surface modification of core-shell particles}

First, for subsequent processing of magnetic silica shell particles (Fig. 1), e.g. for the preparation of elastomeric opal films, their quantitative dispersibility after calcination at $500{ }^{\circ} \mathrm{C}$ is an important prerequisite. In general, surface hydroxyl groups of the silica particles are prone to condensation reactions leading to the formation of inter- and intra-particle $\mathrm{Si}-\mathrm{O}-\mathrm{Si}$ bridges. ${ }^{27}$ Inter-particle condensation reactions lead to irreversible aggregations which make subsequent processing steps for opal film preparation impossible. Furthermore, in the case of vertical deposition and co-assembly methods, aggregations are causing undesired cracks and defects during opal film formation. To overcome these obstacles, we introduce the trimethyl ethoxy silane (TMES) surface modification, which partly saturates the reactive surface hydroxyl groups as depicted in Fig. S1 (ESI $\dagger$ ) and described in the Experimental section.

The first indication of successful surface modification with TMES of the particles could be observed by dispersing the particles in water (Fig. 2). The photographs illustrate the change in the surface polarity of polymer/silica hybrid particles after the modification step: while the pristine silica particles, which were prepared by the Stöber process, are wetted immediately after contact with water and sink (Fig. 2A), the modified particles are not wettable and therefore swim at the water surface (Fig. 2B).

Successful modification of hybrid particles with TMES was additionally monitored by zeta potential measurements (Fig. S2, ESI + ). The shift of measured zeta potential in the positive direction from $-54 \mathrm{mV}$ to $-48 \mathrm{mV}$ after TMES-functionalization confirmed the expectation of a reduced negative charge on the particle surface and is a further evidence for successful silica modification. Even though the methyl-silicon bonding is not stable at $500{ }^{\circ} \mathrm{C}$ - as shown by thermogravimetric analysis (TGA) in an oxygen atmosphere (Fig. S3, ESI $\dagger)^{28}$ - the modification ought to prevent the formation of particle aggregations supporting a convenient dispersibility after thermal treatment. This is very important for transferring (functional) silica shell particles into subsequent reaction steps. As a method of choice for clarifying the positive influence of TMES on inorganic particle modification and hence preventing particle agglomeration, solid-state NMR was used to get a detailed picture of the surface on the molecular level.

In a first step, the ${ }^{13} \mathrm{C}$ cross polarization magic-angle spinning (CP MAS) spectra of both samples, the TMES functionalized

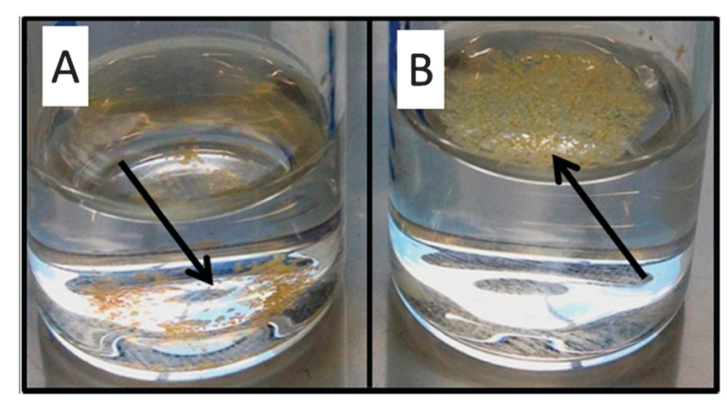

Fig. 2 Pristine particles (A) and TMES-modified particles (B) in water 

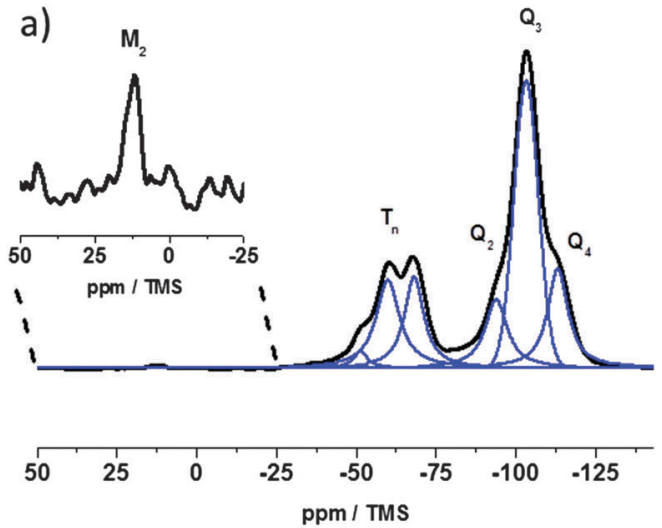

b)
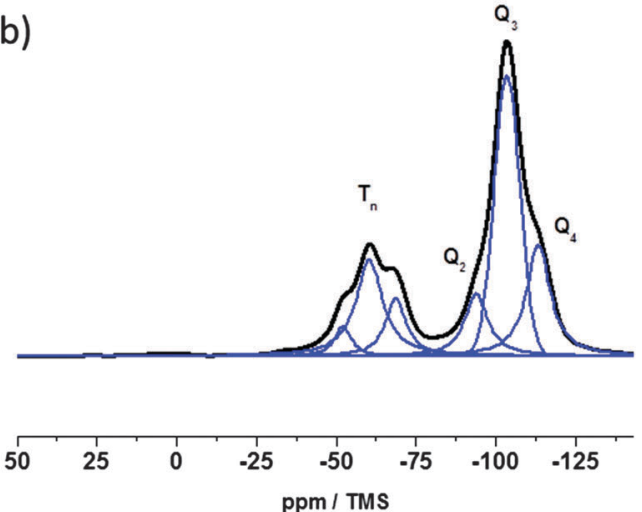

Fig. $3{ }^{29} \mathrm{Si} \mathrm{CP}-\mathrm{MAS}$ spectra measured at $8 \mathrm{kHz}$ spinning with a contact time of $2 \mathrm{~ms}$ and the corresponding deconvolution of these spectra of the TMES-treated sample (a) and the blank Stöber sample (b).

sample and the sample obtained from the Stöber process were recorded. The measured spectra at $11 \mathrm{kHz}$ (Fig. S4, ESI $\dagger$ ) show slight differences for the signals in the aliphatic region. Especially, at around $-1.5 \mathrm{ppm}$ a signal becomes visible in the spectrum of the TMES functionalized sample which indicates the presence of -O-Si- $\left(\mathrm{CH}_{3}\right)_{3}$. In the next step, the ${ }^{29} \mathrm{Si} \mathrm{CP}$ MAS spectra of both samples (Fig. 3) were compared. While the bulk silica phase represented by $Q_{n}$ groups at ca. $-111.5 \mathrm{ppm}\left(Q_{4}\right)$, $-102.5 \mathrm{ppm}\left(Q_{3}\right)$, and $-95 \mathrm{ppm}\left(Q_{2}\right)$ is visible in both samples, ${ }^{29}$ an additional signal is observed at $+\mathbf{1 1 . 5} \mathrm{ppm}$ for the TMES sample which clearly assigns $\mathbf{M}_{2}$ groups which are introduced by the reaction with the TMES reagent. Finally, for both samples signals in the range of $T_{n}$ groups $(-50 \mathrm{ppm}$ to $-70 \mathrm{ppm})$ are visible. For the TMES sample three signals centered at $-68.5 \mathrm{ppm}\left(T_{4}\right),-60.5 \mathrm{ppm}\left(T_{3}\right)$, and $-52 \mathrm{ppm}\left(T_{2}\right)$ while in the case of the Stöber sample three signals at $-68 \mathrm{ppm}\left(T_{4}\right)$, $-61 \mathrm{ppm}\left(T_{3}\right)$ and $-53 \mathrm{ppm}\left(T_{2}\right)$ with different intensities are observed. Note that the assignment of the signals of $T_{n}$ groups and of $\mathrm{M}_{2}$ groups was done according to the work of Albert et al. ${ }^{30}$ The proposed structures are given in the ESI $\dagger$ (Fig. S5). From these results it is evidenced that the reaction with the TMES reagent occurred, and that the organic molecule is covalently linked to silica.

To compare the degree of saturation of $\mathrm{OH}$-groups in both samples quantitatively, in a first step the relaxation time for the
${ }^{29} \mathrm{Si}$ nucleus in the different samples was measured employing a saturation recovery experiment. Taking these $T_{1}$ times (Table S1, $\mathrm{ESI} \dagger$ ) for both samples into account, a direct polarization experiment was performed. The obtained spectra are depicted in the ESI $\dagger$ (Fig. S6, ESI $\dagger$ ). The presence of $Q_{2}$ and $Q_{3}$ groups in both spectra indicated that the $\mathrm{OH}$-groups are not fully functionalized in the TMES-treated sample. This observation is not very surprising since the steric hindrance of the methyl groups of TMES linked on the surface provides a large number of surface OH-groups, which are non-accessible for reaction with the TMES reagent. For TMES-functionalized samples the ratio $\left(Q_{3}+Q_{2}\right) / Q_{4}=0.47$ (areas: $(2.86+2.70) / 11.77=0.47)$ was slightly smaller than for the sample of the pristine particles $\left(Q_{3}+Q_{2}\right) / Q_{4}=0.62$ (areas: $(4.384+1.428) / 9.31=0.62)$ which proves that $\mathrm{Si}-\mathrm{OH}$ groups are partially modified by the TMES reagent. In combination with the results obtained by the CP MAS NMR and zeta potential measurements it can be concluded that TMES reacted with the surface of the particles. Due to the steric hindrance of the trimethylsilyl group only a few TMES molecules are needed to cover the surface, which is consistent with the low intensity of the signals assigning to -O-Si- $\left(\mathrm{CH}_{3}\right)_{3}$ as well as the only slightly reduced intensity of the $Q_{2}$ and $Q_{3}$ in the TMES sample.

The initial goal of TMES-modification was the use of magnetic hollow particles obtained from single source precursors in opal film processing. For this purpose, the influence of TMES on calcination of precursor particles and dispersibility of the resulting magnetic hollow spheres is investigated and described in this section. The samples of both materials, the initial hybrid particles and the TMES-modified particles, were treated in the same manner. Vacuum drying, calcination and dispersion were conducted under exactly the same conditions in order to prove an enhanced dispersibility of calcined particles due to the use of TMES modification. For this purpose, $5 \mathrm{~g}$ of deionized water was added to $10 \mathrm{mg}$ of thermally treated particles and the dispersion samples were compared after $5 \mathrm{~min}$ of ultrasonication. The results are documented via photographs (Fig. S7, ESI $\dagger$ ) and the corresponding TEM images of both particle dispersions (Fig. S8, ESI $\dagger$ ). The photographs indicate an incomplete dispersion of bare particles obtained from the Stöber process (see the arrow in Fig. S7B, ESI $\dagger$ ) compared with a stable dispersion of TMESmodified particles (Fig. S7, ESI, $\dagger$ right). Furthermore, Fig. S7A $(\mathrm{ESI} \dagger)$ clearly shows that both samples could be wetted in water, in contrast to TMES-treated samples before calcination (Fig. 2). This confirms that the nanoparticles are not hydrophobic anymore after calcination. TEM images additionally give evidence for the positive influence of TMES-modification on the particle agglomeration (Fig. S8, ESI $\dagger$ ). By drop-casting a low concentrated dispersion of well-separated particles on a carbon-coated copper grid, a monolayer of respective particles can be found during TEM measurements. This behavior is observed in the case of precursor particles obtained from the Stöber process (Fig. S8A, ESI $\dagger$ ). After thermal treatment and dispersing the particles in water, a significant difference between TMES-modified particles and pure particles is found. While the modification again led to formation of a monolayer (Fig. S9, ESI $\dagger$ ), the blank test revealed a significant amount of agglomerations (Fig. S8C, ESI $\dagger$ ). 
Besides these observations, a deeper insight into the chemical structure of (modified) particles after the calcination step is given by solid state NMR. The absence of $T_{n}$ groups in ${ }^{29} \mathrm{Si}$ CP-MAS (Fig. S10, ESI $\dagger$ ) clearly demonstrates that after heating the sample at $500{ }^{\circ} \mathrm{C}$, all organic compounds decomposed and only the inorganic $\mathrm{SiO}_{2}$ matrix represented by the $Q_{n}$ groups (in the range of $-85 \mathrm{ppm}$ and $-120 \mathrm{ppm}$ ) remained. Finally, another interesting observation was found in the ${ }^{29} \mathrm{Si}$ MAS spectra measured by direct polarization at different spinning rates (Fig. S11, ESI $\dagger$ ). In contrast to the samples prior to calcination, these spectra show spinning sidebands, which are untypical for ${ }^{29} \mathrm{Si}$ of silica materials since the ${ }^{29} \mathrm{Si}$ anisotropy of this nucleus is usually small. Combined with a relatively short $T_{1}$ time of $31.5 \mathrm{~s}$ this is an indication for paramagnetic interactions. Similar observations have been described and summarized by the work of Bakhmutov et al. who investigated the influence of paramagnetic cations such as $\mathrm{Mn}^{2+}$ on the relaxation times of ${ }^{29} \mathrm{Si}$ as well as on the spectral line shape. ${ }^{31,32}$ The authors found reduced $T_{1}$ relaxation times as well as spectra including a large number of spinning sidebands which they refer to interactions of the ${ }^{29} \mathrm{Si}$ with the paramagnetic cation. These observations underline the magnetic behavior of the prepared particles within this study.

\section{Polymer shell formation for magnetic core-shell nanoparticles and opal film formation}

For any of the envisaged opal film formation techniques comprising the vertical deposition as well as the melt-shear organization, the calcined and dispersed particles need to be coated uniformly with a polymer shell. Firstly, seeded starved feed emulsion polymerization as a method of choice is known for the preparation of suitable monodisperse hybrid particles. ${ }^{14,33}$ In order to transfer the silica particles from Stöber conditions to the medium for seeded emulsion polymerization, 3-(trimethoxysilyl)propyl methacrylate (MEMO) as a hydrophobic compound and a grafting group can be used. ${ }^{34}$ For this purpose, particles need to be transferred from ethanol to water by cautious azeotropic distillation, keeping the volume constant, otherwise the particles tend to agglomerate in the highly concentrated dispersion. Again, by using TMES as the functionalizing agent for surface modification instead of MEMO, the particles can easily be vacuum-dried and dispersed in an emulsifier containing water since there are neither trifunctional silanol nor any acrylate groups which are prone to crosslinking reactions (Fig. 4).

Furthermore, the hydrophobic particle surface is capable of forming a polymer shell via seeded emulsion polymerization. This way, a crosslinked poly(allyl methacrylate-co-methyl methacrylate) shell was formed on the modified particles. In general, the polymer shell thickness is adjustable by varying the amount of monomer emulsion feed. An increase from $\sim 250 \mathrm{~nm}$ of the particle diameter for the core particles up to $\sim 490 \mathrm{~nm}$ featuring a polymer shell was intended in this study. The accuracy of this method was demonstrated via TEM images of polymer shell/inorganic core particle dispersion after using the drop-casting method (Fig. 5).

Yolk-shell particles with an inner core which is addressable by an external magnetic field in the context of opal films will be

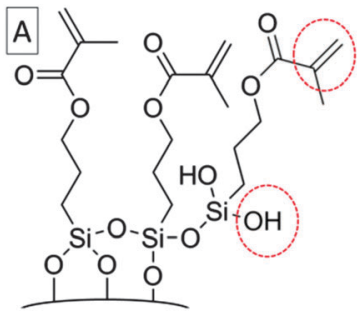

B

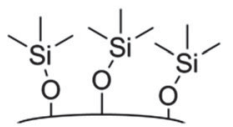

Fig. 4 Segment of the particle surface functionalized with MEMO (A) and TMES (B).

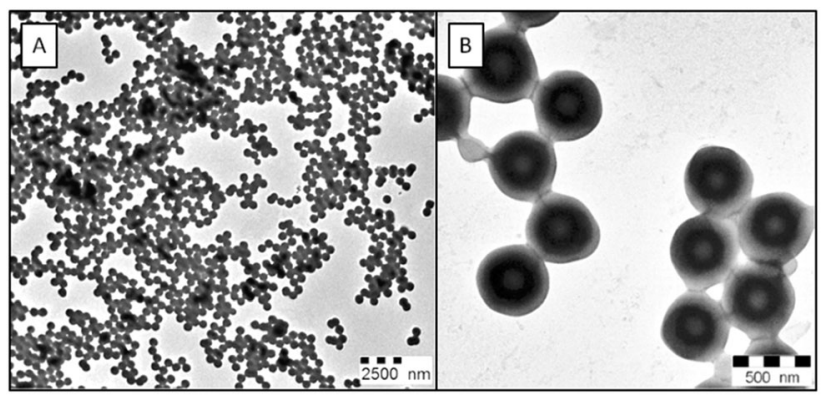

Fig. 5 TEM images of polymer shell/inorganic core particles obtained by seeded starved feed emulsion polymerization starting with magnetic hollow spheres (Fig. S9, ESI $\dagger$ ). The scale bar for the overview TEM image correspond to $2500 \mathrm{~nm}(\mathrm{~A})$ and $500 \mathrm{~nm}$ (B) for the zoom-in TEM image.

of interest for stimuli-responsive optical materials and membranes. Due to this intention, hybrid core-shell particles shown in Fig. 5 were introduced again into a Stöber process in order to form a second silica shell. For this purpose, the precursor particles needed to be heated for polymer decomposition, resulting in air voids between both silica particles featuring different diameters. To ensure a quantitative dispersion of yolk-shell particles for further processing, TMES functionalization was again conducted in this step. The successful preparation of well-defined nanorattle-type particles is evidenced by TEM and SEM images as compiled in Fig. 6. There, the inner spheres correspond to the magnetic nanorattle-type particles, which are inside a hollow silica nanoparticle having larger diameters.

Moreover, the thus easily obtained functional nanoparticles were used for the preparation of more complex architectures, i.e. elastomeric opal films and double-inverse opal films as

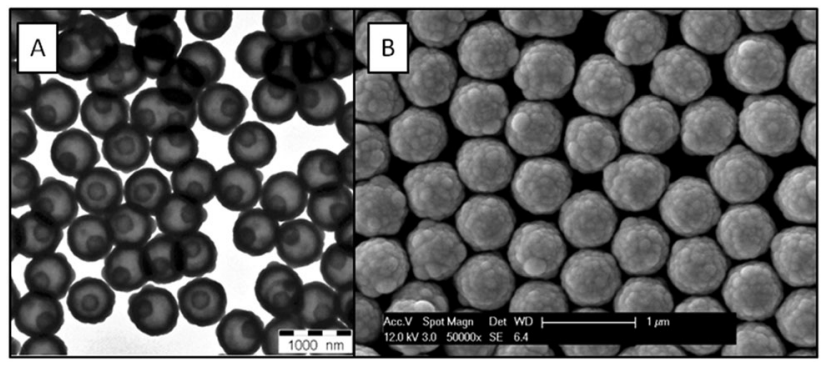

Fig. 6 TEM image (A) and SEM image (B) of yolk-shell particles with the magnetic inner core. 

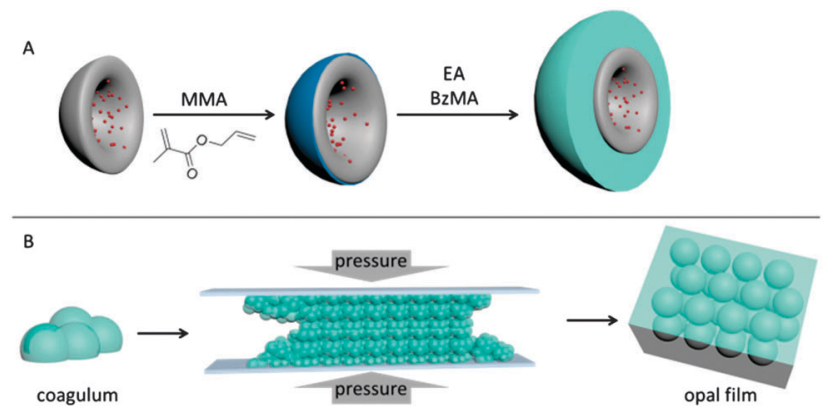

Fig. 7 Formation of a soft and partially grafted polymer shell on thermally treated nanorattle-type particles by emulsion polymerization of methyl methacrylate (MMA) and allyl methacrylate (ALMA) as interlayers in a second, and benzyl methacrylate (BzMA) and ethyl acrylate (EA) as shell materials in the last step (A). The principle of opal film formation via the melt-shearprocess starting from the coagulated polymer mass (B) (refer to text).

described in the ensuing sections proving the usability of this modification method.

For the opal film preparation via the melt-shear organization technique, monodisperse particles with a rigid core and a partially grafted meltable shell are required. This technique and corresponding particle architectures are schematically given in Fig. 7.

A partially grafting of the soft polymer shell is necessary for maintaining the core-shell particle structure during meltshearing. This was guaranteed by the incorporation of allyl methacrylate in an interlayer as previously described..$^{35}$ In the case of calcined hybrid particles, the emulsion polymerization was carried out as depicted in Fig. 7A and described in the Experimental section. Instead of an entirely rigid shell, for this purpose, a slightly crosslinked poly(allyl methacrylate ${ }_{10 \mathrm{wt} \%}-\mathrm{Co}-$ methylmethacrylate $e_{90 w t} \%$ ) interlayer and a partially grafted poly(ethyl acrylate ${ }_{70 \mathrm{wt} \%}-c o$-benzyl methacrylate $\left.{ }_{30 \mathrm{wt} \%}\right)$ shell were formed on calcined particles. The principle of melt-shearing is schematically depicted in Fig. 7B.

First of all, the particles obtained from emulsion polymerization were precipitated in methanol with sodium chloride. After washing and drying, the coagulated mass was mixed with benzophenone as the photo-crosslinker in a micro-extruder and finally, the rubber-like mass was pressed between two planar plates at $140{ }^{\circ} \mathrm{C}$ and 180 bar for $3 \mathrm{~min}$ (see the Experimental section). A photograph of an exemplary opal film disc at two different angles is shown in Fig. 8A.

Angle dependency for the reflection colors according to Bragg's law was demonstrated by UV/Vis reflection spectroscopy measurements (Fig. 8B). The spectra for different angles of incidence ranging from 90 degrees to 30 degrees were plotted in the corresponding figure and revealed that the entire range from red to blue could be covered simply by tilting the film. Besides angle dependency, deformation of the colloidal crystal in the soft polymer matrix also allows the change of reflection color. The application of selective pressure or stretching of the elastomeric opal film leads to a local decrease in lattice distances and thus, reflection color blue shifts. Reflection spectra for stretching experiments are exemplarily shown in Fig. S12 (ESI $\dagger$ ). Since the synthesis strategy of such functional opal films is
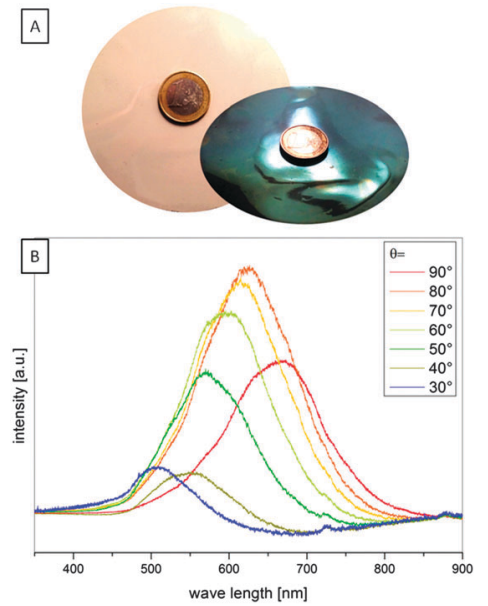

Fig. 8 Photographs of elastomeric opal film at different angles (A), angledependent UV/Vis reflection spectra (B).

expandable to various polymer systems featuring inorganic and silica-modified cores, the transfer to softer polymers or gels is promising for influencing the reflection color by external triggers, e.g. an electrical or magnetic field. The magnetic character of prepared elastomeric opal films having nanorattle-type magnetic core particles were further investigated by magnetic force microscopy as described in the ensuing section.

To verify the magnetic properties of the novel opal films on the nanoscale, magnetic force microscopy (MFM) was applied. An atomic force microscope is capable of detecting magnetic forces when a cantilever coated with a magnetic layer on the tip side is used. To successfully visualize magnetic structures, the long-range magnetic forces need to be separated from other tip-sample forces, e.g. Pauli-repulsive forces or van-der-Waals forces, which are commonly one order of magnitude stronger. Due to the long-range nature of magnetic forces, this can be realized in the lift mode, a two-pass scanning method, where each line is scanned twice. In the first pass, the topography of the sample is measured in the conventional amplitude or frequency modulation mode. In the second pass, the oscillating cantilever is lifted off the surface to a certain height where the influence of short-range forces can be neglected. Here, the same profile acquired in the first pass is equidistantly scanned without amplitude or frequency feedback. Hence, the observables such as the amplitude, frequency or phase are free to "sense" the magnetic interaction between the tip and the surface. Nevertheless, electrostatic forces, if existing, can influence the oscillation of a vibrating cantilever at higher lift heights as well. To separate electrostatic from magnetic forces, we conducted lift mode measurements of the same sample spot using a magnetic cantilever in the demagnetized and magnetized state. For that, we demagnetized a magnetic cantilever (see the Experimental section) and imaged a $5 \times 5 \mu \mathrm{m}^{2}$ area of the magneto-opal film in the lift mode at different lift heights. Fig. 9A and B show the topography scan (first pass) and the phase image in the lift scan (second pass, lift height is $20 \mathrm{~nm}$ ) of the magneto-opal film using the demagnetized cantilever, respectively. The distribution of the 


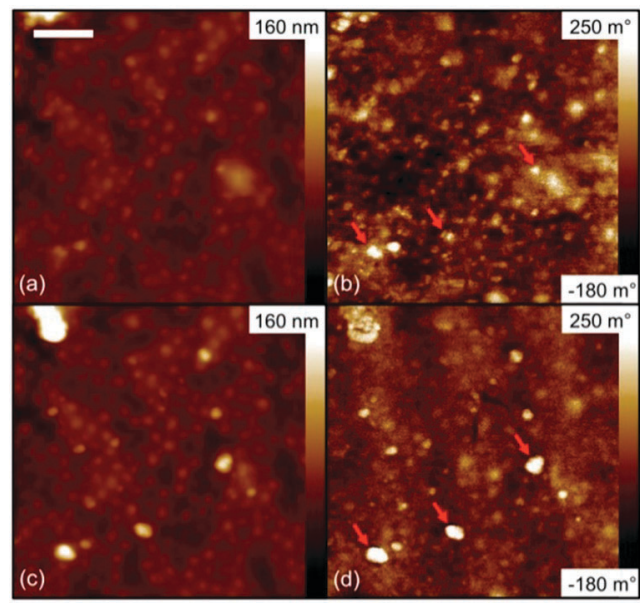

Fig. 9 MFM images of a nanorattle-type opal film using a demagnetized ( $a$ and $b$ ) and a magnetized ( $c$ and d) cobalt coated cantilever. The topography ( $a$ and $c$ ) and the lift mode phase images ( $b$ and $d$ ) clearly reveal the lateral distribution of the magnetic particles in the polymer film. Red arrows highlight particles which clearly show a higher contrast in the lift mode phase image taken by the magnetized cobalt coated cantilever. Scale bar corresponds to $1 \mu \mathrm{m}$.

magnetic particles within the polymer film can be deduced from the topography image since they slightly protrude from the average profile of the top polymer layer. Due to electrostatic forces, however, the particles were clearly visible in the lift scan as bright dots. After magnetizing the cantilever by a permanent magnet and rescanning the same area, the topography image of Fig. 9C revealed the lateral distribution of the magnetic particles more clearly than Fig. 9A. In addition to van-der-Waals and Pauli repulsive forces, magnetic forces were present whenever the tip moved across the lateral position of a particle. This additional force gradient had to be compensated by the $z$-piezo keeping the oscillation amplitude of the cantilever at a constant level leading to different apparent protrusion heights of the particles. ${ }^{36}$ The lift mode image (Fig. 9D) revealed a higher phase contrast of the magnetic particles compared to the contrast visible in the phase image taken by the demagnetized cantilever. Red arrows in Fig. 9 highlight three particles clearly showing this phenomenon.

To explicitly quantify the enhanced image contrast detected by the magnetized cantilever over the contrast measured using the demagnetized cantilever, the phase shift of these three particles with respect to the surrounding area was averaged at different lift heights $(20 \mathrm{~nm}, 40 \mathrm{~nm}, 60 \mathrm{~nm} 80 \mathrm{~nm}$ and $100 \mathrm{~nm})$. Fig. 10 correlates the averaged phase shift values of the three particles in dependence of the lift height for the demagnetized and magnetized cantilever, respectively. The graph unambiguously reveals that with the magnetized cantilever the phase shift detected on the same particles is much higher at each tip-sample lift height than the phase shift measured with the demagnetized cantilever. These results ultimately prove the magnetic nature of the particles in the opal films and the magnetic properties of the nanorattle particle opal films on the nanoscale. However, it can also be concluded that an electrostatic interaction was existent between the oscillating tip and the particles.

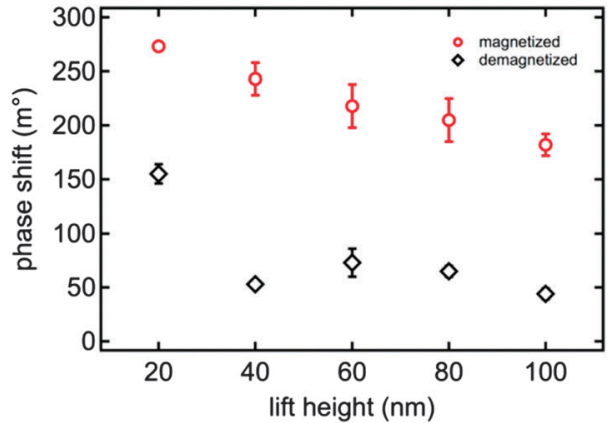

Fig. 10 Averaged phase shift measured on three particles with respect to the surrounding area versus lift height. The red circles correspond to the phase shift values detected using the magnetized cantilever/tip whereas the black diamonds correspond to the phase shift values measured using the cantilever in the demagnetized state.

As another proof of the herein established chemical modification concept, elastomeric opal films featuring the novel yolk-shell particles in a polymer matrix were prepared. For this purpose, the transfer of yolk-shell particles shown in Fig. 6 to seeded emulsion polymerization of ethylacrylate (EA) was conducted as described in the Experimental section. The formation of the polymer shell as well as subsequent processing steps (washing, drying, extrusion, and pressing) was conducted as described in the Experimental section. A photograph of the prepared elastomeric film is shown in Fig. 11A. SEM images of the film surface (Fig. S13A, ESI $\dagger$ ) and a cross-section (Fig. S13B, $\mathrm{ESI} \dagger$ ) confirmed the successful incorporation of monodisperse yolk-shell particles in a polymer matrix, evidencing a 3D colloidal crystal structure. The absence of visible reflection colors in this particular case basically arises from the particle size in combination with low refractive index contrast. The reflection wavelength would be far in the infra-red range (see Table S2 for calculations and used values, ESI $\dagger$ ).

Colvin et al. developed the method of vertical deposition, ${ }^{37}$ wherein the solvent of a colloidal dispersion is slowly evaporated inside a narrow vessel. Inside the vessel, a glass substrate is vertically deposited allowing the spheres to crystallize successively onto the substrate. This route was significantly improved by Aizenberg et al. These authors developed an elegant method of vertical deposition enabling the generation of compact composite

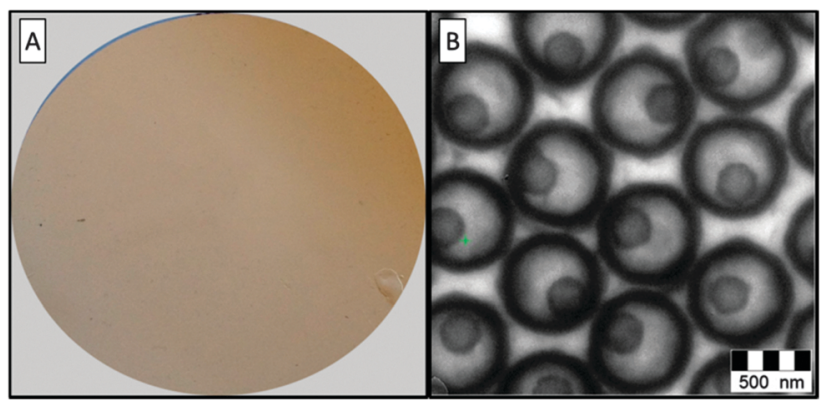

Fig. 11 Photograph of the prepared elastomeric film (A) and the TEM image of yolk/shell particles with a soft polymer shell via the drop cast method of aqueous dispersion (B) 

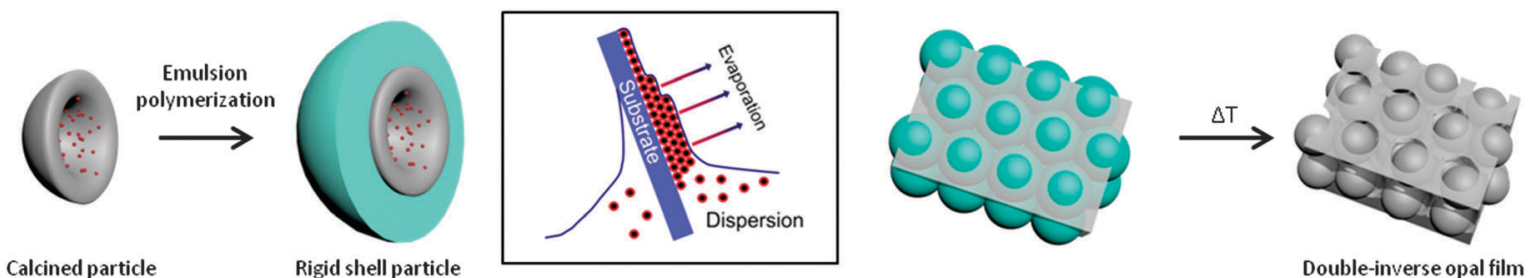

Fig. 12 Simplified scheme for the magnetic hollow particle modification by emulsion polymerization (left) and the vertical deposition method (middle, see text). The opal films obtained by this method can be infiltrated with different sol-gel precursors, which yield particle-nested double inverse opal films after thermal treatment (right).

colloidal crystals via co-assembly of polymeric colloidal spheres and a silica sol-gel precursor solution. ${ }^{11,38}$

Within the present study, using the hybrid particles shown in Fig. 5, these methods lead to double-inverse opal films. For this purpose, again, the improved dispersibility due to TMES modification is important to minimize defect structures due to possible aggregation phenomena during particle deposition. The preparation route for double-inverse opal films starting from calcined hybrid particles via vertical deposition and co-assembly is depicted in Fig. 12.

As a prerequisite for almost defect-free double-inverse opal films, the formation of monodisperse core/shell hybrid particles has already succeeded (compare Fig. 5). Tetraethoxyorthosilicate (TEOS) as the $\mathrm{SiO}_{2}$-precursor and titanium(rv)-bis-lactato-bisammonium dihydroxide (TiBALDH) as the $\mathrm{TiO}_{2}$-precursor were used for the preparation of double-inverse opal films. To prevent cracks all over the film and to keep the film free from a cover layer, the optimum ratio of precursors to particles needed to be identified. Photographs and SEM images of prepared doubleinverse opal films are shown in Fig. 13.

We were able to prepare double-inverse opal films with rather large crack-free areas (Fig. S14, ESI $\dagger$ ). These films revealed brilliant and angle-dependent reflection colors (Fig. 13C, reflection spectra are shown in Fig. S15, ESI $\dagger$ ). SEM images in Fig. 13 clearly show that the film preparation leads to nested particles

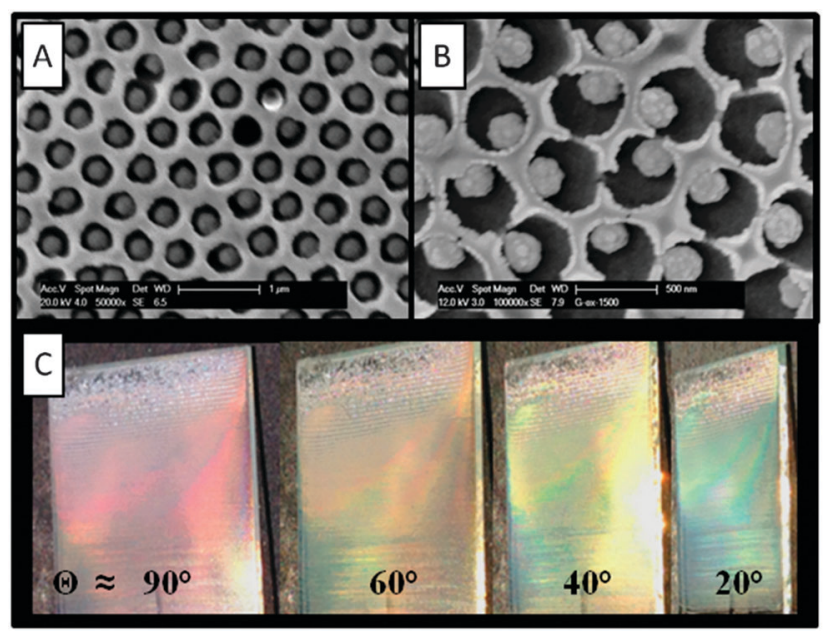

Fig. 13 SEM image of double-inverse opal film with the silica matrix $(A)$ and the titania matrix (B). Photographs of double-inverse opal film at different angles (C). Scale bars are $1 \mu \mathrm{m}$ in (A) and $500 \mathrm{~nm}$ in (B). from the inner $\mathrm{SiO}_{2}$ core in a silica matrix (A) as well as a titania matrix (B).

The here described synthesis strategy leads to double-inverse opal films with a magnetic inner particle whereas the matrix material is variable and the space between the matrix and the inner particle is tuneable by varying the thickness of the polymer shell.

\section{Conclusions}

Within this study, the preparation of complex nanoparticle architectures, i.e. magnetic nanorattle-type particles and their 3-dimensional colloidal crystal structures were described. As a key step for the presented routes comprising successive chemical steps and particle organization techniques, a convenient surface modification for nanoparticles featuring a silica shell was highlighted. For this purpose, trimethyl ethoxy silane (TMES) for surface modification was used as a chemical protection layer prior to thermal treatment of the metallopolymer particle precursors. This modification was shown to be useful to avoid agglomeration phenomena. TMES treatment was additionally applied for the formation of a hydrophobic particle shell for the formation of well-defined core shell particles by transfer into the emulsion polymerization. Successful surface modification with TMES was evidenced by zeta potential measurements, while solid state NMR allowed gaining deeper insights into the chemical structure and molecular character of the binding site of modified nanoparticles. Successful transfer of the magnetic nanorattle-type particles from the applied Stöber process to emulsion polymerization provided access to complex opal film architectures. By application of the melt-shear organization technique and the vertical deposition method for the modified core-shell particles the formation of 3D colloidal structures with excellent optical properties was possible. The herein described TMES-modification strategy is expected to be universal for the silica surface, which will pave the way for smart optical materials with stimulus-responsive behavior based on complex hierarchical nanoparticle architectures.

\section{Materials and methods}

\section{Reagents}

All chemicals and solvents were purchased from Fisher Scientific, Sigma-Aldrich and Alfa Aesar and used as received if not otherwise stated. The water soluble azoinitiator VA-086 was donated by 
Wako Chemicals GmbH. 2-(Methacryloyloxy)-ethyl ferrocene carboxylate (FcMA) was synthesized according to the literature. ${ }^{39}$ The removal of the methyl methacrylate (MMA) stabilizer was achieved by extraction with $1 \mathrm{M}$ sodium hydroxide solution. Afterwards, MMA was washed with water until neutrality, followed by drying with sodium sulfate.

\section{Synthesis of seed particles}

A $1 \mathrm{~L}$ vessel equipped with a stirrer, a reflux condenser and nitrogen feeding was heated to $75{ }^{\circ} \mathrm{C}$ and then filled with monomer emulsion containing $180 \mathrm{mg}$ of sodium dodecylsulfate (SDS), $434 \mathrm{~g}$ of deionized water, $7.2 \mathrm{~g}$ of destabilized methyl methacrylate (MMA) and $0.8 \mathrm{~g}$ of allyl methacrylate (ALMA). The polymerization was initiated by adding $20 \mathrm{mg}$ of sodium disulfite (NaDS), $150 \mathrm{mg}$ of sodium persulfate (NaPS) and $50 \mathrm{mg}$ of NaDS in this order (each component is dissolved in $5 \mathrm{~mL}$ of water). The polymerization ran for $5 \mathrm{~h}$ at $75{ }^{\circ} \mathrm{C}$ before the dispersion was cooled down to ambient temperature for storage and further use.

\section{Synthesis of cross-linked PMMA-co-PFcMA particles}

2-(Methacryloyloxy)-ethyl ferrocenecarboxylate (FcMA) containing particles were synthesized by starved feed emulsion polymerization. A $250 \mathrm{~mL}$ vessel equipped with a stirrer, a reflux condenser and nitrogen feeding was filled with $75 \mathrm{~g}$ of the above mentioned seed particle dispersion and $30.5 \mathrm{~g}$ deionized water and then heated to $88{ }^{\circ} \mathrm{C} .10 \mathrm{~min}$ after addition of $250 \mathrm{mg}$ of VA-086 in $10 \mathrm{~g}$ of water, a monomer emulsion containing $110 \mathrm{mg}$ of SDS, $110 \mathrm{mg}$ of Dowfax 2A1, $45 \mathrm{~g}$ of water, $28.5 \mathrm{~g}$ of MMA, $13.9 \mathrm{~g}$ of FcMA and $4.6 \mathrm{~g}$ of ALMA was added at a constant flow rate of $0.2 \mathrm{~mL} \mathrm{~min}{ }^{-1}$. After complete addition of monomer emulsion, the reaction was finished by holding the temperature for an additional hour.

\section{Synthesis of $\mathrm{SiO}_{2} @ F c M A-c o-M M A$ particles}

In a typical procedure, $20 \mathrm{~g}$ of FcMA-containing dispersion (22.7 wt\%) and $9.2 \mathrm{~g}$ water were filled in a $50 \mathrm{~mL}$ vessel with a stirrer, a reflux condenser and nitrogen feeding. The mixture was stirred at $88{ }^{\circ} \mathrm{C}$ and buffered at $\mathrm{pH} 7-7.5$ using $0.8 \mathrm{~mL}$ of a sodium dihydrogen phosphate ( $2 \%$ aqueous) and sodium bicarbonate ( $2 \%$ aqueous) buffer. After adding $50 \mathrm{mg}$ of VA-086, a monomer emulsion with $0.12 \mathrm{~g}$ of ALMA, $1.2 \mathrm{~g}$ of MMA, $2.4 \mathrm{~g}$ of 3-(trimethoxysilyl)propyl methacrylate, $10 \mathrm{mg}$ of SDS, $2.5 \mathrm{~g}$ of water, $0.18 \mathrm{~mL}$ of buffer solution was fed at a constant flow rate of $50 \mu \mathrm{L} \mathrm{min}{ }^{-1}$. After complete addition of monomer emulsion, the reaction was finished by holding the temperature for $30 \mathrm{~min}$. In a subsequent step, the particles were transferred in a Stöber process. Typically, $31.5 \mathrm{~g}$ of latex was mixed with $840 \mathrm{~mL}$ of ethanol and $48 \mathrm{~g}$ of ammonia solution (25 vol\%). Then $30 \mathrm{~g}$ of tetraethoxyorthosilicate (TEOS) diluted in $30 \mathrm{~mL}$ of ethanol were added and the dispersion was gently stirred at ambient temperature for $16 \mathrm{~h}$.

\section{TMES functionalization for the silica shell nanoparticles}

For TMES functionalization, $20 \mathrm{~g}$ of dispersion of silica/polymer hybrid particles obtained from the previous step was treated with $0.7 \mathrm{~mL}$ of ethoxytrimethylsilane at ambient temperature and heated to $60{ }^{\circ} \mathrm{C}$ for one hour. Excess of TMES and solvent was evaporated in a vacuum. After drying, the obtained hybrid particles in vacuum were tempered at $500{ }^{\circ} \mathrm{C}$ for $5 \mathrm{~h}$.

\section{Elastomeric opal film preparation}

Analogous to particles from the Stöber process, $4 \mathrm{~g}$ of magnetic hollow spheres were dispersed in ammoniacal ethanol and hydrophobized by TMES functionalization. After drying, the particles were dispersed in $70 \mathrm{~g}$ of water with $10 \mathrm{mg}$ of Dowfax $2 \mathrm{~A} 1$ by ultrasonication. The dispersion was filled in a $250 \mathrm{ml}$ vessel with a stirrer, a reflux condenser and nitrogen feeding and heated up to $75{ }^{\circ} \mathrm{C}$. After addition of $30 \mathrm{mg}$ of NaPS in $3 \mathrm{~g}$ of water, the following emulsions were added with a constant flow

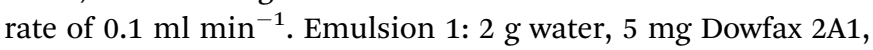
$0.3 \mathrm{~g}$ MMA, $0.03 \mathrm{~g}$ ALMA, $0.01 \mathrm{~g}$ MEMO. Emulsion 2: $9.5 \mathrm{~g}$ water, $5 \mathrm{mg}$ Dowfax 2A1, 0.9 MMA, $0.1 \mathrm{~g}$ ALMA. Emulsion 3: $23 \mathrm{~g}$ water, $15 \mathrm{mg}$ Dowfax 2A1, $4.9 \mathrm{~g}$ ethyl acrylate (EA), and $2.1 \mathrm{~g}$ benzyl methacrylate (BzMA). After complete addition of monomer emulsion, the reaction was finished by maintaining the temperature for an additional hour.

For the preparation of elastomeric opal films, the obtained core/shell particles were precipitated in methanol, containing a small amount of saturated sodium chloride solution. The precipitate was filtered, water washed and dried under reduced pressure. In a microextruder (microl, DSM Research) at $130{ }^{\circ} \mathrm{C}$, the resulting elastomeric mass was compacted and (optionally) mixed with benzophenone ( $3 \mathrm{wt} \%$ ). A $3 \mathrm{~g}$ portion of the rubbery polymer was covered with a PET film and heated to $140{ }^{\circ} \mathrm{C}$ between the plates of a Collin laboratory press. Melt flow was induced by applying 180 bar for $3 \mathrm{~min}$, resulting in an elastomeric opal film of about $15 \mathrm{~cm}$ in diameter.

\section{Double-inverse opal film preparation}

Magnetic hollow spheres were treated as described for elastomeric opal films. Only instead of the EA/BzMA emulsion, a MMA/ALMA emulsion was added. The amount of emulsion is dependent on the desired shell thickness.

Vertical deposition was conducted as described in the literature. ${ }^{11}$ Typically, in a $30 \mathrm{ml}$ beaker $1 \mathrm{~g}$ of a $2.5 \mathrm{wt} \%$ dispersion was added to $20 \mathrm{~g}$ of water and $0.2 \mathrm{~g}$ of a $1 \mathrm{~h}$ stirring solution of TEOS, $0.1 \mathrm{M} \mathrm{HCl}$ and ethanol $(2: 2: 3)-$ in the case of the $\mathrm{TiO}_{2}$ matrix, $0.2 \mathrm{~g}$ of a $50 \%$ TiBALDH-solution is used instead. Then, a glass slide is vertically positioned in the beaker and water evaporates within two days at $65{ }^{\circ} \mathrm{C}$ in an oven. The polymeric component is removed by heat treating the glass slide for $2 \mathrm{~h}$ at $500{ }^{\circ} \mathrm{C}$.

\section{Yolk-shell particle synthesis}

A second silica shell on silica/polymer particles is formed analogous to the first silica shell, described above. This includes TMES functionalization, calcination and elastomeric film formation (with an EA shell instead of EAcoBzMA).

\section{Instrumentation}

All solid-state NMR spectra were recorded at room temperature on a Bruker Avance III HD 600 spectrometer equipped with a $4 \mathrm{~mm}$ broad band $\mathrm{H} / \mathrm{X}$ probe. ${ }^{1} \mathrm{H}$ spectra were recorded at $600.12 \mathrm{MHz}$, 
${ }^{13} \mathrm{C}$ spectra at $150.92 \mathrm{MHz}$ and ${ }^{29} \mathrm{Si}$ spectra at $119.22 \mathrm{MHz}$. All spectra were referenced to TMS employing adamantane as external standard for ${ }^{13} \mathrm{C}(38.5 \mathrm{ppm})$ and TSP as external standard for ${ }^{1} \mathrm{H}(0 \mathrm{ppm})$ and ${ }^{29} \mathrm{Si}(0 \mathrm{ppm}) .{ }^{13} \mathrm{C}$ CP-MAS spectra were recorded utilizing ramped CP-MAS sequences ${ }^{40}$ at a spinning rate of $11 \mathrm{kHz}$ employing a contact time of $2 \mathrm{~ms}$ and a recycle delay of $4 \mathrm{~s} .{ }^{29} \mathrm{Si} \mathrm{CP-MAS}$ spectra were recorded at $8 \mathrm{kHz}$ spinning employing a contact time of $2 \mathrm{~ms}$ and a recycle delay of $2 \mathrm{~s}$. Tppm decoupling $^{41}$ was applied during data acquisition. $T_{1}$ times for ${ }^{29} \mathrm{Si}$ were measured employing a saturation recovery experiment with a $90^{\circ}$ saturation pulse train containing $3.5 \mu$ s pulses and a pulse spacing of $5 \mathrm{~ms}$ to ensure saturation of the $z$-magnetization. According to the calculated $T_{1}$ times for quantitative NMR experiments single pulse excitation with a $1.5 \mu$ s excitation pulse was performed employing a recyle delay of $3 \times T_{1}$ to ensure full built up of the signal for quantification.

Zeta potential measurements were performed on a Zetasizer Nano ZS (Malvern Instruments Ltd, UK).

TEM experiments were carried out on a Zeiss EM 10 electron microscope operating at $60 \mathrm{kV}$. All shown images were recorded using a slow-scan CCD camera obtained from TRS (Tröndle) in bright field mode. Camera control was computer-aided using the ImageSP software from TRS.

For UV/Vis measurements a deuterium/tungsten halogen lamp (HL-2000, Ocean optics) was used. Angle-dependent reflection measurements were carried out using a custom-built goniometer setup. For calcination, the particles were treated in a Nabertherm LT 5/11 high temperature oven. TGA was performed on a Mettler TGA 1 from $30{ }^{\circ} \mathrm{C}$ to $700{ }^{\circ} \mathrm{C}$ with $10 \mathrm{~K} \mathrm{~min}^{-1}$. For the AFM experiments, a Dimension Icon atomic force microscope (Bruker AXS, Santa Barbara, CA) was used for acquiring the lift mode images. Topography imaging was accomplished in the amplitude modulation mode. $5 \times 5 \mu^{2}$ areas were scanned with a scan rate of $0.5-1.5 \mathrm{~Hz}$ and a lateral resolution of $512 \times 512$ pixel. Cobalt coated cantilevers of the type PPPMFM were purchased from Nanoandmore (Wetzlar, Germany). The fundamental resonant frequency and the quality factor of the cantilever in air were $f_{0}=75 \mathrm{kHz}$ and $Q=265$, respectively. The force constant of the cantilever $k=3.5 \mathrm{~N} \mathrm{~m}^{-1}$ was measured by the thermal noise method. ${ }^{42}$ For the demagnetization, the cantilever was heated in a furnace (THERMCONCEPT, Bremen, Germany) to $1200{ }^{\circ} \mathrm{C}$, well-above the Curie temperature of cobalt $\left(1120{ }^{\circ} \mathrm{C}\right) .{ }^{43}$ The cantilever was then kept heated at $1200{ }^{\circ} \mathrm{C}$ for $5 \mathrm{~min}$ before slowly cooling down to room temperature (approx. within $3 \mathrm{~h}$ ) by switching off the furnace. For the re-magnetization, the cantilever was mounted on top of a standard permanent magnet for $3 \mathrm{~min}$. It is worth-mentioning that no external magnetic field was applied to the samples. Thus, the magnetic stray field of the cantilever only magnetized the particles at the sample surface. Topography images were first-order flattened to correct for sample tilt and thermal drift during imaging. Phase images were zero-order flattened excluding the particle areas in order to set the background phase to zero. To estimate the phase shift of the particles, we drew averaged cross-sectional profiles across the particles over an area of $200 \times 20 \mathrm{~nm}^{2}$ each.

\section{Acknowledgements}

The authors want to thank Johannes Klitsch for energetic support in synthetic work and Andreas Geißler for zeta potential measurements. Further acknowledgements apply to Wako Chemicals $\mathrm{GmbH}$ for donating the VA-086 initiator and we thank Prof. G. Buntkowsky (TU Darmstadt) for generous allocations of measurement time to perform the multinuclear solid-state NMR experiments. The authors cordially thank Prof. M. Biesalski and his group (Macromolecular Chemistry and Paper Chemistry Department, TU Darmstadt) for analytical support. M. G. acknowledges the "Fonds der Chemischen Industrie" and the Max-Buchner Foundation (Dechema).

\section{Notes and references}

1 K. R. Phillips, G. T. England, S. Sunny, E. Shirman, T. Shirman, N. Vogel and J. Aizenberg, Chem. Soc. Rev., 2016, 45, 281-322.

2 N. Zhao, Z. Wang, C. Cai, H. Shen, F. Liang, D. Wang, C. Wang, T. Zhu, J. Guo, Y. Wang, X. Liu, C. Duan, H. Wang, Y. Mao, X. Jia, H. Dong, X. Zhang and J. Xu, Adv. Mater., 2014, 26, 6994-7017.

3 J. Wang, Y. Zhang, S. Wang, Y. Song and L. Jiang, Acc. Chem. Res., 2011, 44, 405-415.

4 Y. Zhao, Z. Xie, H. Gu, C. Zhu and Z. Gu, Chem. Soc. Rev., 2012, 41, 3297-3317.

5 R. O. Prum, R. Torres, C. Kovach, S. Williamson and S. M. Goodman, J. Exp. Biol., 1999, 202, 3507-3522.

6 N. Kumano, T. Seki, M. Ishii, H. Nakamura and Y. Takeoka, Angew. Chem., Int. Ed., 2011, 50, 4012-4015.

7 K. R. Phillips, N. Vogel, Y. Hu, M. Kolle, C. C. Perry and J. Aizenberg, Chem. Mater., 2014, 1622-1628.

8 K. Rhee do, B. Jung, Y. H. Kim, S. J. Yeo, S. J. Choi, A. Rauf, S. Han, G. R. Yi, D. Lee and P. J. Yoo, ACS Appl. Mater. Interfaces, 2014, 6, 9950-9954.

9 J. Kim, S. A. Bencherif, W. A. Li and D. J. Mooney, Macromol. Rapid Commun., 2014, 35, 1578-1586.

10 M. Retsch and U. Jonas, Adv. Funct. Mater., 2013, 23, 5381-5389.

11 B. Hatton, L. Mishchenko, S. Davis, K. H. Sandhage and J. Aizenberg, Proc. Natl. Acad. Sci. U. S. A., 2010, 107, 10354-10359.

12 Y. Takeoka, J. Mater. Chem. C, 2013, 1, 6059-6074.

13 C. Fenzl, T. Hirsch and O. S. Wolfbeis, Angew. Chem., Int. Ed., 2014, 53, 3318-3335.

14 C. G. Schäfer, M. Gallei, J. T. Zahn, J. Engelhardt, G. P. Hellmann and M. Rehahn, Chem. Mater., 2013, 25, 2309-2318.

15 D. Scheid, C. Lederle, S. Vowinkel, C. G. Schäfer, B. Stühn and M. Gallei, J. Mater. Chem. C, 2014, 2, 2583-2590.

16 J. Ge and Y. Yin, Angew. Chem., Int. Ed., 2011, 50, 1492-1522. 17 C. G. Schäfer, T. Winter, S. Heidt, C. Dietz, T. Ding, J. J. Baumberg and M. Gallei, J. Mater. Chem. C, 2015, 3, 2204-2214.

18 I. Lee, D. Kim, J. Kal, H. Baek, D. Kwak, D. Go, E. Kim, C. Kang, J. Chung, Y. Jang, S. Ji, J. Joo and Y. Kang, Adv. Mater., 2010, 22, 4973-4977.

19 X. Xu, G. Friedman, K. D. Humfeld, S. A. Majetich and S. A. Asher, Chem. Mater., 2002, 14, 1249-1256. 
$20 \mathrm{H} . \mathrm{Hu}, \mathrm{H}$. Zhong, C. Chen and Q. Chen, J. Mater. Chem. C, 2014, 2, 3695.

21 J. Ge, J. Goebl, L. He, Z. Lu and Y. Yin, Adv. Mater., 2009, 21, 4259-4264.

22 J. Ge and Y. Yin, J. Mater. Chem., 2008, 18, 5041-5045.

23 J. Ge, S. Kwon and Y. Yin, J. Mater. Chem., 2010, $20,5777$.

24 J. Ge, L. He, Y. Hu and Y. Yin, Nanoscale, 2011, 3, 177-183.

25 D. Scheid, G. Cherkashinin, E. Ionescu and M. Gallei, Langmuir, 2014, 30, 1204-1209.

26 S. Vowinkel, C. G. Schäfer, G. Cherkashinin, C. Fasel, F. Roth, N. Liu, C. Dietz, E. Ionescu and M. Gallei, J. Mater. Chem. C, 2016, DOI: 10.1039/C5TC03483C.

27 O. W. Flörke, H. A. Graetsch, F. Brunk, L. Benda, S. Paschen, H. E. Bergna, W. O. Roberts, W. A. Welsh, C. Libanati, M. Ettlinger, D. Kerner, M. Maier, W. Meon, R. Schmoll, H. Gies and D. Schiffmann, Silica, Wiley-VCH Verlag GmbH \& Co. KGaA, Weinheim, Germany, 2008.

28 J. McElwee, R. Helmy and A. Y. Fadeev, J. Colloid Interface Sci., 2005, 285, 551-556.

29 A. Grünberg, Y. P. Xu, H. Breitzke and G. Buntkowsky, Chem. - Eur. J., 2010, 16, 6993-6998.

30 K. Albert and E. Bayer, J. Chromatogr., 1991, 544, 345-370.

31 V. I. Bakhmutov, Chem. Rev., 2011, 111, 530-562.
32 V. I. Bakhmutov, B. G. Shpeizer and A. Clearfield, Magn. Reson. Chem., 2006, 44, 985-988.

33 C. G. Schäfer, S. Vowinkel, G. P. Hellmann, T. Herdt, C. Contiu, J. J. Schneider and M. Gallei, J. Mater. Chem. C, 2014, 2, 7960.

34 C. G. Schäfer, C. Lederle, K. Zentel, B. Stuhn and M. Gallei, Macromol. Rapid Commun., 2014, 35, 1852-1860.

35 C. G. Schäfer, B. Viel, G. P. Hellmann, M. Rehahn and M. Gallei, ACS Appl. Mater. Interfaces, 2013, 5, 10623-10632.

36 C. Dietz, E. T. Herruzo, J. R. Lozano and R. Garcia, Nanotechnology, 2011, 22, 125708.

37 P. Jiang, J. F. Bertone, K. S. Hwang and V. L. Colvin, Chem. Mater., 1999, 11, 2132-2140.

38 Y. Vasquez, M. Kolle, L. Mishchenko, B. D. Hatton and J. Aizenberg, ACS Photonics, 2014, 1, 53-60.

39 M. Mazurowski, M. Gallei, J. Li, H. Didzoleit, B. Stühn and M. Rehahn, Macromolecules, 2012, 45, 8970-8981.

40 G. Metz, X. L. Wu and S. O. Smith, J. Magn. Reson., Ser. A, 1994, 110, 219-227.

41 A. E. Bennett, C. M. Rienstra, M. Auger, K. V. Lakshmi and R. G. Griffin, J. Chem. Phys., 1995, 103, 6951-6958.

42 H. J. Butt and M. Jaschke, Nanotechnology, 1995, 6, 1-7.

43 B. Chatterjee, Thin Solid Films, 1977, 41, 227-233. 